\title{
Versatile Soft Robot Gripper Enabled by Stiffness and Adhesion Tuning via Thermoplastic Composite
}

\author{
Ryan Coulson, ${ }^{1}$ Christopher J. Stabile, ${ }^{2}$ Kevin T. Turner, ${ }^{2}$ and Carmel Majidi ${ }^{1,3}$
}

\begin{abstract}
Within the field of robotics, stiffness tuning technologies have potential for a variety of applications-perhaps most notably for robotic grasping. Many stiffness tuning grippers have been developed that can grasp fragile or irregularly shaped objects without causing damage and while still accommodating large loads. In addition to limiting gripper deformation when lifting an object, increasing gripper stiffness after contact formation improves load sharing at the interface and enhances adhesion. In this study, we present a novel stiffness and adhesion tuning gripper, enabled by the thermally induced phase change of a thermoplastic composite material embedded within a silicone contact pad. The gripper operates by bringing the pad into contact with an object while in its heated, soft state, and then allowing the pad to cool and stiffen to form a strong adhesive bond before lifting the object. Pull-off tests conducted using the gripper show that transitioning from a soft to stiff state during grasping enables up to $6 \times$ increase in adhesion strength. Additionally, a finite element model is developed to simulate the behavior of the gripper. Finally, pick-and-place demonstrations are performed, which highlight the gripper's ability to delicately grasp objects of various shapes, sizes, and weights.
\end{abstract}

Keywords: soft gripper, stiffness modulation, adhesion-based grasping, thermoplastic composite, tunable adhesion

\section{Introduction}

$\mathbf{S}$ TIFFNESS TUNING - THE ABILITY of a material or system to transition between a soft conformable state and a rigid load-bearing state-is an aspect of robotics that has received considerable attention in recent years. This is especially true within the field of soft robotics, where many different approaches have been developed to enable stiffness tuning for various applications. ${ }^{1-3}$ Generally, these approaches can be grouped into one of four different categories: (1) granular jamming $^{4-8}$; (2) electro- and magnetorheological materials ${ }^{9,10}$; (3) shape memory polymers ${ }^{11-17}$; and (4) low melting point materials, for example, waxes, ${ }^{18,19}$ polymers, ${ }^{20-27}$ and metal alloys. ${ }^{28-31}$ While the references provided above are generally relevant to this article, they represent only a fraction of all the research on stiffness tuning for soft robotics; for a more thorough review, consult other sources. ${ }^{1-3}$

The work presented in this article focuses on the use of a low melting point polymer (LMPP) for robotic grasping applications. The stiffness tuning principle of LMPPs is simple: these materials are stiff at room temperature (Young's modulus $>10 \mathrm{MPa}$ ), and they soften as their temperature increases, ultimately undergoing a phase change that results in a soft "melt" state. Previously, several robotic grippers have been developed using LMPPs as an enabling material, including multifingered grippers with stiffness tuning ligaments or joints made of conductive propylene-based elastomer $(\mathrm{cPBE})^{25}$ and acrylate-based thermoplastic polymer, ${ }^{20}$ as well as silicone posts with stiffness tuning cores made of $\mathrm{cPBE},{ }^{26}$ which enabled tunable adhesion. However, while these grippers demonstrated the potential of LMPPs to aid in robotic grasping, their ability to effectively grasp a wide range of objects was limited. The goal of this research was to introduce an LMPPbased materials architecture and gripper design that allows for more versatile soft robot grasping.

The gripper described in this article (Fig. 1) achieves tunable adhesion by controlling the stiffness of a "contact pad," consisting of a stiffness tuning element embedded within a silicone matrix, similarly to the device developed by Tatari et al. ${ }^{26}$ During grasping, the gripper is able to establish

\footnotetext{
${ }^{1}$ Robotics Institute, Carnegie Mellon University, Pittsburgh, Pennsylvania, USA.

${ }^{2}$ Department of Mechanical Engineering and Applied Mechanics, University of Pennsylvania, Philadelphia, Pennsylvania, USA.

${ }^{3}$ Department of Mechanical Engineering, Carnegie Mellon University, Pittsburgh, Pennsylvania, USA.
} 

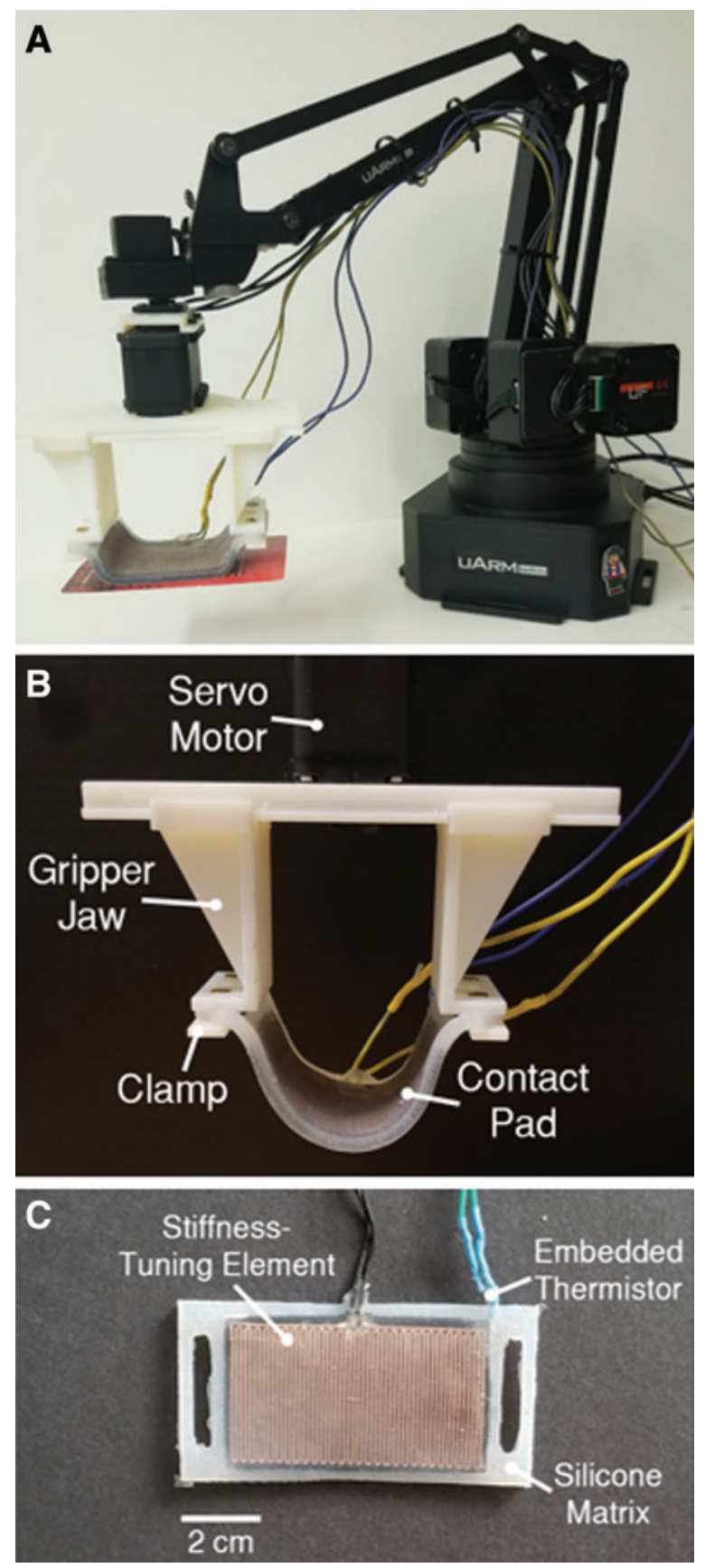

FIG. 1. (A) Fully integrated robotic system, with gripper mounted to robot arm. (B) Gripper with contact pad attached. (C) Contact pad containing a stiffness tuning element composed of a carbon-filled PCL composite and serpentine copper heater. PCL, polycaprolactone. Color images are available online.

large contact areas by bringing the pad into contact with objects while the pad is in its soft conformable state. Before lifting an object, the contact pad is stiffened, which enables improved load sharing at the contact interface, and therefore, enhanced adhesion, as shown by Tatari et al. ${ }^{26}$ Thus, the stiffness tuning capabilities of the gripper enable two of the primary factors required for strong adhesive bonding: large contact areas and a high level of load sharing at the contact interface. When the gripper is required to release an object, it simply returns the contact pad to its soft state, leading to reduced load sharing and accordingly reduced adhesion strength.

The general adhesion mechanism that this gripper relies upon is "dry adhesion," which is enabled by the van der Waals forces that form when its silicone exterior comes into contact with other solid objects. ${ }^{32}$ A number of other robotic grippers have been developed that rely upon dry adhesion, many of which are inspired by the fibrillar structure of gecko's feet. ${ }^{26,33-35}$ However, except for the stiffness tuning posts developed by Tatari et al., ${ }^{26}$ none of these grippers leverage stiffness change to control adhesion strength. Additionally, our gripper is distinct from the gripper developed by Tatari et al. due to its ability to conform to a variety of surface geometries. We also note that various robotic grippers have been developed that rely upon other adhesion-based mechanisms, including electroadhesion, ${ }^{36}$ tackiness-based adhesion, ${ }^{37}$ and adhesion induced by magnetic fields. ${ }^{38}$ For a more comprehensive review of adhesion-based grasping, the reader should consult other sources. ${ }^{3,39}$

In the following sections, we investigate the hypothesis that an LMPP-based stiffness tuning element can be used in a soft robotic gripper to enable adhesion-based grasping that is both adaptive (meaning the geometry of the gripper can match the geometry of the object being grasped) and controllable (meaning the force capacity of the gripper can be modulated). In adhesion-based grasping, the maximum adhesion force (also referred to as pull-off force) between the gripper and a target object determines the load capacity of the gripper. As a result, pull-off force is often used as a metric for evaluating the efficacy of adhesion-based grippers. ${ }^{18,34,38,40,41}$ To test the effectiveness of our gripper and help guide its future design, we conduct experiments to measure pull-off force as a function of various parameters. Additionally, a finite element model is developed to simulate the behavior of the gripper. Finally, the gripper is used for pick-and-place demonstrations with various objects.

\section{Methodology}

\section{Material selection, synthesis, and characterization}

The LMPP chosen as the stiffness tuning element for this gripper was a thermoplastic composite consisting of polycaprolactone (PCL) loaded with carbon black. The process for fabricating the composite is described in detail by Rich et $a{ }^{27}$ Qualitative testing revealed that the most favorable composition was an 80:20 ratio of PCL (Perstorp AB) to carbon black (Alfa Aesar) by weight. The selected PCL had a molecular weight of $88.4 \mathrm{~kg} / \mathrm{mol}$ (CAPA 6800).

Qualitative testing indicated that as the composite's loading fraction of carbon black increases, its stiffness at room temperature increases while its ductility decreases. Similarly, reductions in the molecular weight of PCL lead to increased stiffness and reduced ductility. These results are consistent with those reported in a detailed characterization by Rich et $a l^{27}$ We desired a composite that was maximally stiff at room temperature without being susceptible to fracture, and we found that the composite described above met these requirements. An additional consideration was the viscosity of the composite in its melt state. Our tests showed that viscosity increases with molecular weight of the polymer and loading fraction of carbon black (also consistent with results reported by Rich $e a_{l}{ }^{27}$ ). We required that the viscosity would be high 


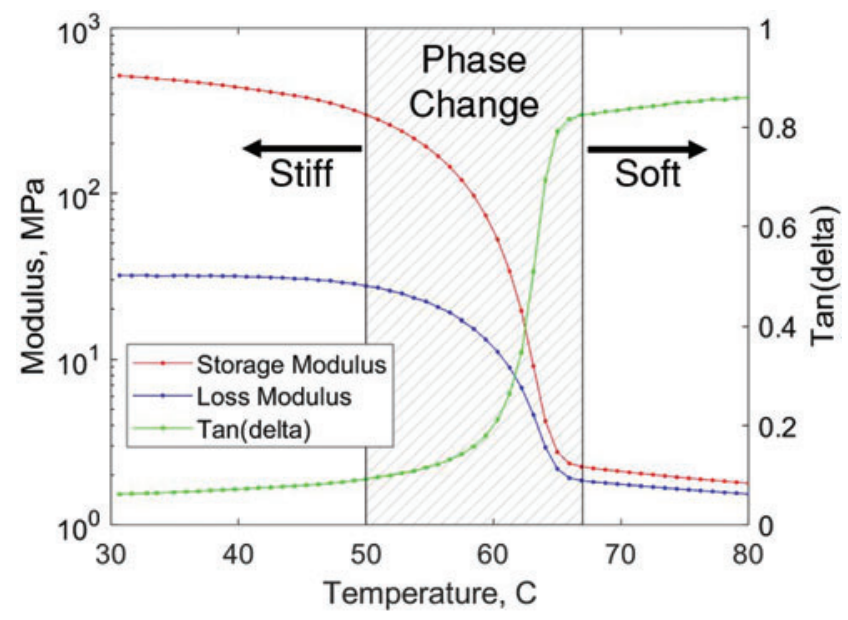

FIG. 2. Results from DMA test of PCL composite with PCL of molecular weight $88.4 \mathrm{~kg} / \mathrm{mol}$ and $20 \%$ loading fraction of carbon black. DMA, dynamic mechanical analysis. Color images are available online.

enough that the composite would maintain structure in its melt state to support the embedded heater (described in subsequent sections). This requirement was also satisfied by the selected composition.

To characterize the stiffness tuning capabilities of the composite, we conducted a dynamic mechanical analysis (DMA) test with tensile loading. Results are shown in Figure 2. Testing revealed that the composite is mechanically stiff below $\sim 50^{\circ} \mathrm{C}$, undergoes a phase change between $50^{\circ} \mathrm{C}$ and $67^{\circ} \mathrm{C}$, and is soft above $\sim 67^{\circ} \mathrm{C}$. Here, the storage modulus serves as a proxy for the Young modulus. The maximum switching ratio (i.e., the ratio of the moduli between stiff and soft states) for the composite is $\sim 300 \times$, which is sufficiently high for most stiffness tuning applications. ${ }^{1,2}$

\section{Gripper design}

The composite is loaded with carbon black, making it electrically conductive. Therefore, it is possible to heat the composite via Joule heating by attaching electrodes and running electric current directly through the composite. ${ }^{27}$ An ability to heat the composite is necessary because increasing temperature enables phase change. However, during preliminary testing, we found this method of direct Joule heating to be unreliable. The primary issue with direct Joule heating was that any variations in the cross section of the composite would result in the formation of "hot spots," or areas of localized heating. This is because the resistance of the composite at any location is inversely proportional to its cross-sectional area. Thus, in locations where the cross-sectional area was smaller than the nominal cross-sectional area, the resistance of the composite was high, resulting in a greater generation of thermal energy for the same amount of current. The problem with this localized heating was that it would not allow the composite to bend uniformly when subjected to external forces and therefore did not meet the requirements for our application.

To apply uniform heat to the composite, we developed flexible resistance heaters by cutting serpentine copper traces, following the process established by Bartlett et al. ${ }^{42}$ and Markvicka et al. ${ }^{43}$ This process is depicted in Figure 3A. First, a $70 \mu \mathrm{m}$ thick layer of flexible copper-clad polyimide (Pyralux FR8510R; DuPont) was laminated onto a polydimethylsiloxane (PDMS) bed (10:1 Sylgard 184; Dow Corning) with a rigid aluminum backing and cut using an ultraviolet laser (ProtoLaser U3; LPKF), as described in the

A

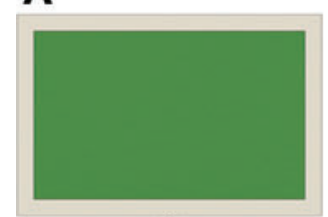

(i)

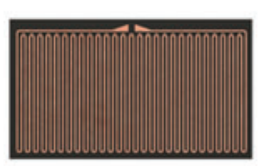

(iv)

Polyimide PCL Composite

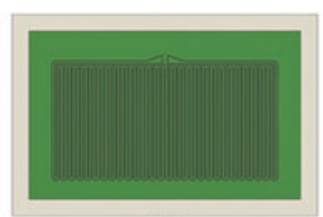

(ii)

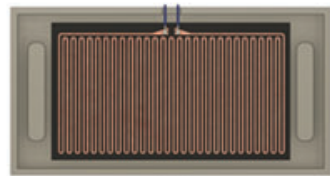

(v)

PDDS VeroWhite

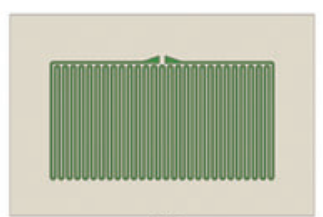

(iii)

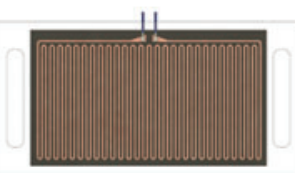

(vi)

Copper Dragon Skin 10
B

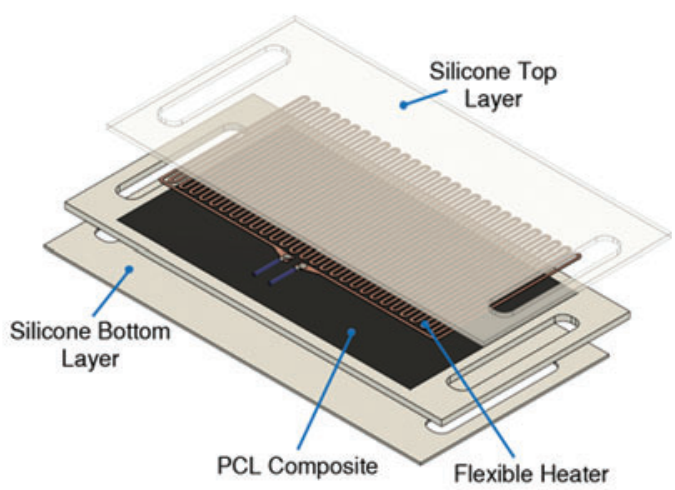

FIG. 3. (A) Fabrication process: (i) Copper-clad polyimide is laminated to a PDMS bed, with polyimide facing upward. (ii) Copper-clad polyimide is machined by ultraviolet laser. (iii) Excess copper-clad polyimide is removed from the PDMS bed, leaving only the serpentine traces. Traces are then laminated to VHB tape and removed from the bed. (iv) Traces and VHB tape (i.e., flexible heater) are placed on top of the composite, with copper facing upward, and adhered to the surface using a hand iron. Wires are then soldered to connection points on the traces. (v) Stiffness tuning element is placed inside the mold, on top of a $500 \mu \mathrm{m}$ thick layer of silicone. Mold is filled with silicone and placed in a $70^{\circ} \mathrm{C}$ oven to cure for $30 \mathrm{~min}$. (vi) Mold is removed from the oven and the contact pad is separated from the mold. (B) Exploded view of contact pad. Thickness of the bottom layer of silicone is $500 \mu \mathrm{m}$. Thickness of the composite is $1.5 \mathrm{~mm}$. Thickness of the top layer of silicone is $1 \mathrm{~mm}$. Total thickness of the contact pad is $3 \mathrm{~mm}$. PDMS, polydimethylsiloxane. Color images are available online. 
study of Markvicka et al. ${ }^{43}$ The width of the resulting traces was $0.6 \mathrm{~mm}$. Then, a $51 \mu \mathrm{m}$ thick layer of adhesive transfer tape (VHB F9460PC; 3M) was laminated to the traces, and the traces and tape were pulled away from the PDMS bed as a single unit, which we refer to as a flexible heater. The heater was then placed on top of a $70 \times 40 \mathrm{~mm}, 1.5 \mathrm{~mm}$ thick rectangular piece of PCL composite, and was adhered to the composite using a hand iron. Thus, by running electric current through the heater's copper traces, heat is generated, which is then distributed through the composite via thermal conduction. The result is that the entire composite can be heated to a nearly uniform temperature. Together, the flexible heater and PCL composite form the stiffness tuning element of the gripper.

After soldering wires to the flexible heater, the stiffness tuning element is embedded in a silicone matrix. The result is a "contact pad," as shown in Figures 1C and 3B. The first step of this process is creating a primary mold for the silicone matrix, as shown in Figure 3A. This mold is made of VeroWhite (Stratasys) and is fabricated using an Objet 3D printer (Stratasys). A separate mold is also fabricated using the same material, which enables casting of a $70 \times 40 \mathrm{~mm}$ layer of silicone (Dragon Skin 10; Smooth-On) with a thickness of $500 \mu \mathrm{m}$. This layer of silicone is placed at the bottom of the primary mold and serves as the interface between the contact pad and target objects. It is important that this layer is cast in a dedicated mold to ensure consistent thickness since its thickness will impact the overall adhesion strength of the contact pad. ${ }^{26}$ Next, a thin layer of uncured silicone (Dragon Skin 10) is poured into the mold, and the stiffness tuning element is placed on top and pressed into contact with the cured layer of silicone beneath. Pouring uncured silicone into the mold before inserting the stiffness tuning element helps to ensure that any cavities on the bottom surface of the composite are filled with silicone, which prevents the formation of air pockets between the composite and the silicone matrix. Finally, the rest of the mold is filled with silicone, and the mold is placed in an oven set to $70^{\circ} \mathrm{C}$ and left to cure for $30 \mathrm{~min}$. The mold is then removed from the oven, and the contact pad is separated from the mold and left to cool.

Embedding the stiffness tuning element in a silicone matrix is important for several reasons. First, the silicone matrix helps the stiffness tuning element to maintain its structure. This is necessary because the PCL composite does not exhibit an elastic restoring force in its soft state. A helpful analogy is that the consistency of the composite in its soft state is similar to that of peanut butter. Thus, any deformation to the composite will be maintained in the absence of external forces. This characteristic is problematic because uniform heating of the composite is dependent on the composite maintaining its original dimensions. Embedding the stiffness tuning element in a silicone matrix solves this problem since the original shape can be restored by the elasticity of the silicone. An additional benefit of the silicone matrix is that it allows for the stiffness tuning element to be easily attached to an external structure. Finally, the silicone matrix allows for greater adhesion than could be achieved with just the composite since the particular silicone used (i.e., Dragon Skin 10) is softer than the composite and therefore allows for more conformal contact.

To control the temperature of the contact pad, a thermistor (10 k $\Omega$ NTC; Uxcell) was embedded between the silicone matrix and the stiffness tuning element (Fig. 1C). This was achieved by cutting a slit in the silicone matrix, then inserting the thermistor and sealing the slit with a flexible adhesive (Sil-Poxy; Smooth-On). The thermistor is used to measure the temperature of the stiffness tuning element, and the temperature is used as feedback for a proportional controller run by a microcontroller (Arduino Uno). The proportional controller restricts the flow of current to the flexible heater to maintain a desired setpoint temperature. Although the temperature at which the composite reaches its soft state is $T_{\text {soft }} \approx 67^{\circ} \mathrm{C}$ (as shown in Fig. 2), testing revealed that the thermistor reading at which this transition occurred was $T_{\text {soft }} \approx 48^{\circ} \mathrm{C}$. So, the setpoint of the proportional controller was established at $50^{\circ} \mathrm{C}$. Correspondingly, it was determined that the thermistor reading at which the composite stiffens is $T_{\text {stiff }} \approx 35^{\circ} \mathrm{C}$.

The time scale of the transition between the stiff and soft states of the contact pad was determined by running a heating test three times. The test started with the contact pad in its stiff state at room temperature $\left(21^{\circ} \mathrm{C}\right)$. Power was then supplied to the heater at $9.0 \mathrm{~V}$, drawing $\sim 1.9 \mathrm{~A}(17 \mathrm{~W})$, and the contact pad was heated until it reached its setpoint temperature $T_{\text {set }}=$ $50^{\circ} \mathrm{C}$. Then, power was removed from the heater, and the pad was allowed to passively cool until the thermistor reading returned to room temperature. During these tests, the average time required for the contact pad to heat from room temperature to its setpoint temperature was $3.3 \mathrm{~min}\left(\dot{T}_{\text {heat }}=0.15^{\circ} \mathrm{C} / \mathrm{s}\right)$. The average time required to cool from the setpoint temperature to $\tilde{T}_{\text {stiff }} \approx 35^{\circ} \mathrm{C}$ was $4.5 \mathrm{~min}\left(\dot{T}_{\mathrm{cool}}=0.055^{\circ} \mathrm{C} / \mathrm{s}\right)$.

The effectiveness of the contact pad in adhering to objects of various shapes and sizes is dependent on its ability to conform to surfaces in its soft state. So, when designing a gripper with an integrated contact pad, the priority was to enable the contact pad to conform to objects with minimal constraints. The result was the design shown in Figure 1B. This gripper is a modified version of the PhantomX Parallel AX-12 Gripper (Interbotix). The parts were fabricated from VeroWhite using an Objet 3D printer. The primary modification to the original gripper design was the addition of fastening structures on the gripper jaws, which could be used to secure the contact pad to the gripper. The gripper was then mounted to a four degree of freedom (DOF) robotic arm (uArm; UFactory), as shown in Figure 1A.

This gripper works by modulating the distance between its jaws when the contact pad is in its soft state. At the beginning of a grasp cycle, the contact pad is in its default, flat shape. As the contact pad transitions to its soft state, it sags slightly due to gravity. Moving the gripper jaws toward each other causes the pad to buckle and deform toward the ground. The robot arm then moves the gripper downward until the pad is brought into contact with the target object. Once sufficient contact has been established between the pad and the object, the gripper stops moving and power is removed from the flexible heater. The contact pad is then allowed to cool until it reaches its stiff state, at which point the gripper is lifted back up with the object in its grasp.

\section{Experimental setup}

To evaluate the effectiveness of the gripper, pull-off tests ${ }^{18,34,38,41}$ were conducted using a materials testing machine (Instron 5969). During the tests, the gripper was attached to $\mathrm{a} \pm 10 \mathrm{~N}$ load cell that was mounted to the crosshead of the Instron, and an acrylic substrate was fixed in place directly 
below. Each individual test started with the contact pad in its stiff state and flat shape. The pad was then heated to $T_{\text {set }}=50^{\circ} \mathrm{C}$, and the gripper jaws were moved toward each other to cause the pad to buckle, resulting in a curved shape. In this curved shape, the contact pad is deflected downward, with the bottom several millimeters above the surface of the acrylic substrate. Next, the Instron crosshead was lowered at $0.167 \mathrm{~mm} / \mathrm{s}$ until reaching a predetermined height. The contact pad was then allowed to rest on the substrate for $5 \mathrm{~min}$ before the Instron crosshead began retracting upward at $0.167 \mathrm{~mm} / \mathrm{s}$. The test ended when the crosshead returned to its starting height. During the test, the Instron records force measurements from the load cell, as well as time stamps and the position of the crosshead. This testing procedure is depicted in Figure 4A and B. Footage of the testing procedure is also available in Supplementary Video S1 and S2. One concern with the testing procedure was the potential accumulation of dust or dirt on the gripper's surface, which could cause a decrease in adhesion strength. ${ }^{44}$ As a result, the contact pad and acrylic substrate were cleaned before each trial to ensure consistent adhesion across testing.

Throughout pull-off testing, several parameters were chosen to be varied systematically. These parameters included the geometry and state of the contact pad, and the geometry of the acrylic substrate. The geometry of the contact pad was controlled by varying the distance between the gripper jaws and the resting height of the Instron crosshead. These parameters are represented by the variables $S$ and $H$, respectively, as depicted in Figure 4D. Note that, when varying $S$ and $H$, the contact area between the contact pad and the substrate does not remain constant. The state of the contact pad before retraction (Fig. 4A, B, Step 5) was either state $=$ soft or state $=$ stiff. In the case of state $=$ soft, the contact pad was kept at $\tilde{T}_{\text {set }}=50^{\circ} \mathrm{C}$ throughout the entire trial. In the case of state $=$ stiff, power was removed from the flexible heater after the Instron crosshead stopped moving downward (Fig. 4A, Step 3). The contact pad was then allowed to cool during the $5 \mathrm{~min}$ resting period, such that its temperature was below $T_{\text {stiff }} \approx 35^{\circ} \mathrm{C}$ once the crosshead began pulling upward. The geometry of the acrylic substrate was varied between flat and cylindrical. A minimum three tests were conducted for each unique parameter combination.

\section{Finite element model}

To gain insight into the mechanics of the gripper, a finite element model was developed to simulate its deformation
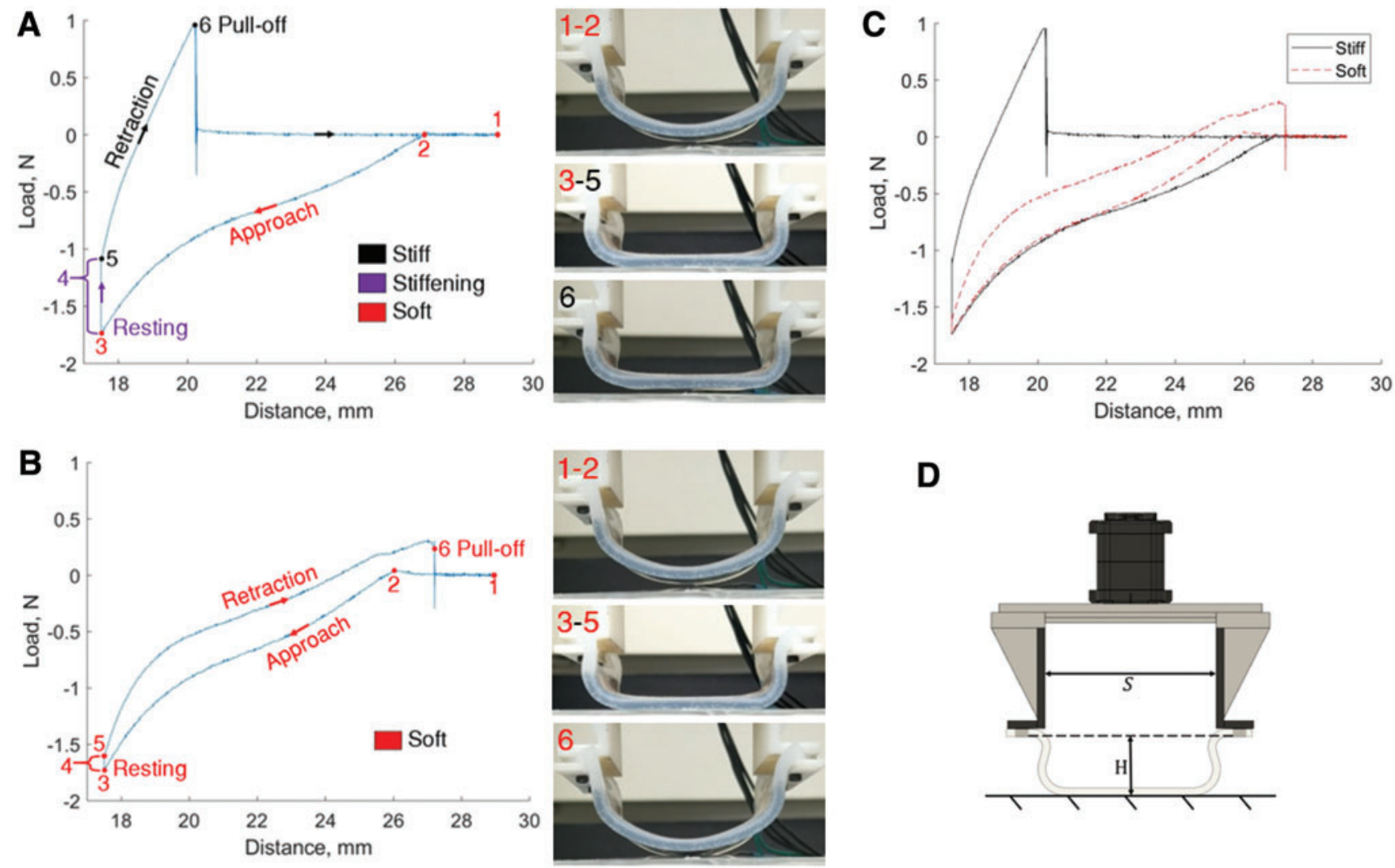

FIG. 4. (A) Pull-off testing procedure for state= stiff: (1) Initially, the contact pad is in its soft state, with the bottom edge several millimeters above the acrylic substrate. Then, the Instron crosshead begins moving downward. (2) Contact pad comes into contact with the substrate. (3) The crosshead reaches its resting height and stops moving. (4) Contact pad is allowed to rest on the substrate for $5 \mathrm{~min}$. During this time, stress relaxation occurs due to the viscoelasticity of the composite. (5) Crosshead begins retracting. (6) The pad loses contact with the substrate. Afterward, the crosshead continues moving upward until it reaches its starting height. (B) Pull-off testing procedure for state $=$ soft. This is identical to the stiff state procedure except for heating of the contact pad. The plots and images shown correspond to tests with parameters, $S=46 \mathrm{~mm}$ and $H=17.5 \mathrm{~mm}$ on a flat substrate. (C) State $=$ stiff and state $=$ soft loading cycles overlaid. (D) Parameters controlling contact pad geometry. $S$ is the distance between the gripper's jaws and $H$ is the distance between the bottom of the gripper jaws and the top of the acrylic substrate. Color images are available online. 
and adhesion. Finite element analysis (FEA) was conducted using ABAQUS/Standard (ABAQUS 2018). The system was modeled as a two-dimensional beam under plane strain conditions and consisting of a stiffness-tunable core between two soft outer layers, as shown in Figure 5A. The initial length of the beam is $S_{0}^{*}=98 \mathrm{~mm}$ and the thickness of the bottom, center, and top layers are $t_{1}=0.5 \mathrm{~mm}, t_{2}=1.5 \mathrm{~mm}$, and $t_{3}=1 \mathrm{~mm}$, respectively. Here, $S_{0}^{*}$ corresponds to the sum of the initial parallel jaw separation $S$, as shown in Figure 4D, and double the distance between the location of applied clamping force and the corresponding inside parallel jaw surface. The soft outer layers were assumed to be linear elastic with Young's modulus $E_{\mathrm{s}}=0.09 \mathrm{MPa}$, Poisson's ratio $v_{\mathrm{s}}=0.49$, and density $\rho_{\mathrm{s}}=1.07 \mathrm{~g} / \mathrm{cm}^{3}$, representative of Dragon Skin 10. The core was assumed to be linear elastic with Young's modulus and Poisson's ratio defined as $E_{\mathrm{c}}{ }^{\text {soft }}=2 \mathrm{MPa}$ and $v_{\mathrm{c}}^{\text {soft }}=0.49$ for the soft state and $E_{\mathrm{c}}{ }^{\text {stiff }}=500 \mathrm{MPa}$ and $v_{\mathrm{c}}{ }^{\text {stiff }}=0.45$ for the stiff state, and constant density $\rho_{c}=1.145 \mathrm{~g} / \mathrm{cm}^{3}$. The material properties used for the core are representative of PCL, with Young's moduli corresponding to the soft and stiff state storage moduli of the PCL composite that was characterized through DMA, as shown in Figure 2.

Simulations were conducted using a structured mesh consisting of plane strain eight-node hybrid biquadratic elements $(\mathrm{CPE} 8 \mathrm{H})$. Only half of the beam was modeled, with a symmetry condition along $x_{1}=0$ and a clamped condition along $x_{1}=S_{0}^{*} / 2$, as shown in Figure 5A, meaning that the edge nodes displace uniformly. Additionally, a surface-to-surface contact interaction with a "hard" contact pressure-overclosure relationship and frictionless behavior was specified between the beam and an analytical rigid surface. The simulations were

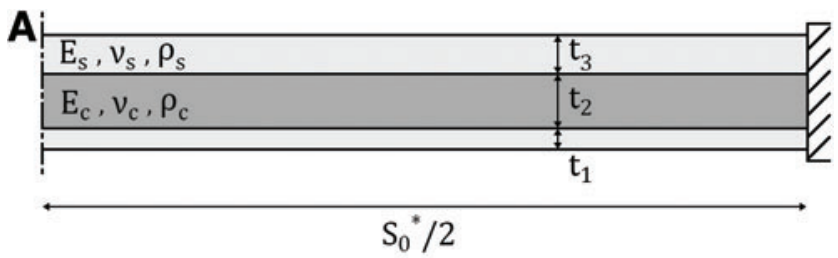

B

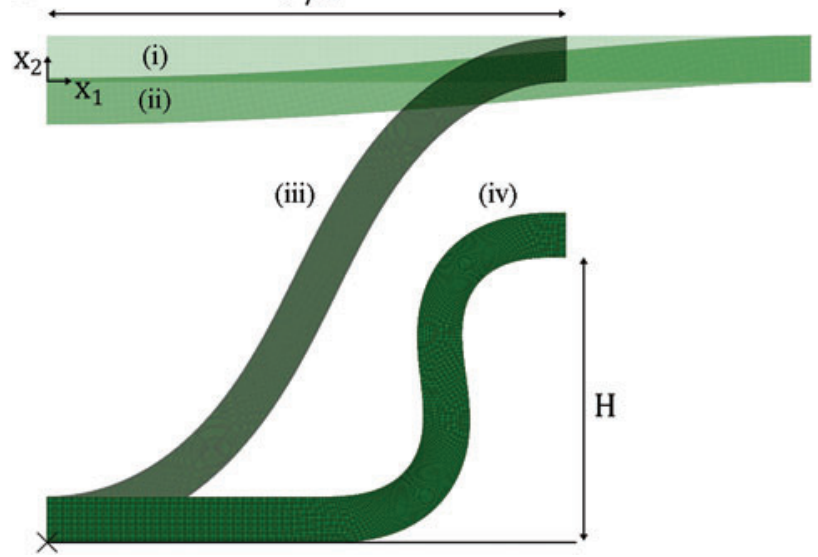

FIG. 5. (A) Finite element schematic of the initial flat state. (B) Finite element deformation process showing (i) the initial flat state, (ii) application of gravity, (iii) compression, and (iv) contact formation. Color images are available online. conducted in three stages consisting of (1) deformation and contact formation, (2) soft-to-stiff transition, and (3) retraction.

During the first stage of the simulations, the beam was deformed and brought into contact with an analytical rigid surface through displacement of the clamped edge. This process involved four substages including (1) initial flat state, (2) application of gravity, (3) compression, and (4) contact formation. The complete deformation process is depicted in Figure 5B. First, the displacement components of the clamped edge were fixed and gravity was activated with a gravitational acceleration $g=9.807 \mathrm{~m} / \mathrm{s}^{2}$. The application of gravity was necessary to introduce an asymmetry in the beam deflection so that it would buckle in the appropriate direction. Second, the clamped edge was displaced inward to induce buckling while establishing the desired parallel jaw separation $S$. Third, with an analytical rigid surface positioned into point contact with the buckled beam, the clamped edge was displaced downward to form the contact while establishing the desired clamping height $H$. Subsequently, the normal displacement of the nodes in contact was fixed with a displacement boundary condition, allowing for the removal of the specified contact interaction.

After achieving the deformed configuration corresponding to the desired values of $S$ and $H$, the material properties of the core were changed from $E_{\text {soft }}$ and $v_{\text {soft }}$ to $E_{\text {stiff }}$ and $v_{\text {stiff. }}$ Meanwhile, the reference configuration of the core elements was set equal to the current/deformed configuration to effectively remove the existing strain.

For the final stage of the simulations, the clamped edge was displaced upward by some small $\delta$ and the corresponding load-displacement curve was used to determine the compliance of the system. This was performed both before and after stiffening the core to evaluate results for state $=$ soft as well as state $=$ stiff, respectively. For an elastic layer adhered to a rigid substrate, the normal adhesion force capacity scales with the critical energy release rate $G_{\mathrm{c}}$, contact area $A$, and system compliance $C,{ }^{45}$ expressed as:

$$
F_{\mathrm{c}} \propto \sqrt{\frac{G_{\mathrm{c}} A}{C}} .
$$

This general scaling law allows for an estimate of the ratio of stiff to soft pull-off forces for a set of $S$ and $H$ values through an assessment of the change in system compliance. While $G_{\mathrm{c}}$ depends on the surface energies of the contacting surfaces, the value remains constant between the soft and stiff states since the outer Dragon Skin layer remains unchanged. Furthermore, while $A$ varies with $S$ and $H$, it remains constant between the soft and stiff states for a given set of $S$ and $H$ values. Therefore, by considering the ratio of stiff to soft pulloff forces, we avoid the need to assume a value for $G_{\mathrm{c}}$, and the effect of modulating $C$ through stiffening the core can be examined directly.

\section{Results and Discussion}

\section{Pull-off testing}

Results from pull-off testing are shown in Figures 6 and 7. For convenience, we consider results from tests with a flat substrate separately from those with a cylindrical substrate. 

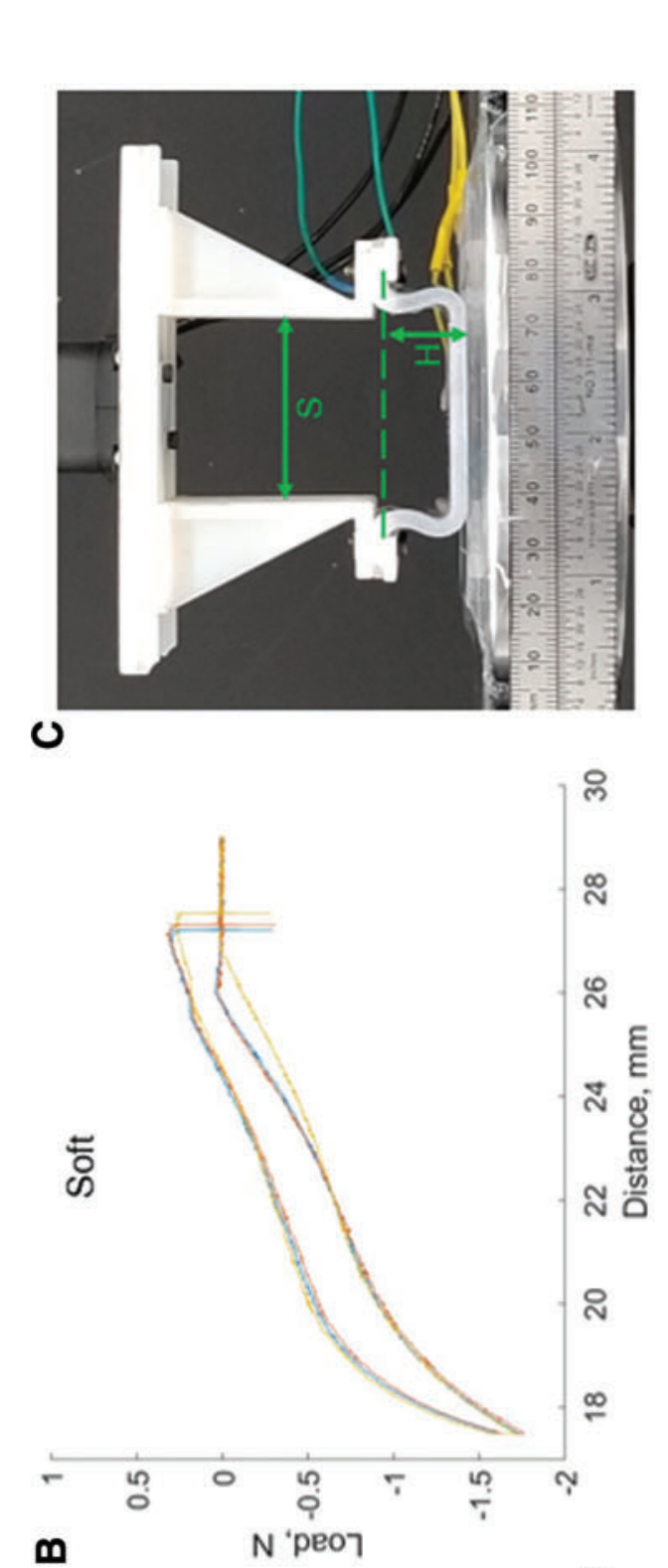

$\boldsymbol{m}$
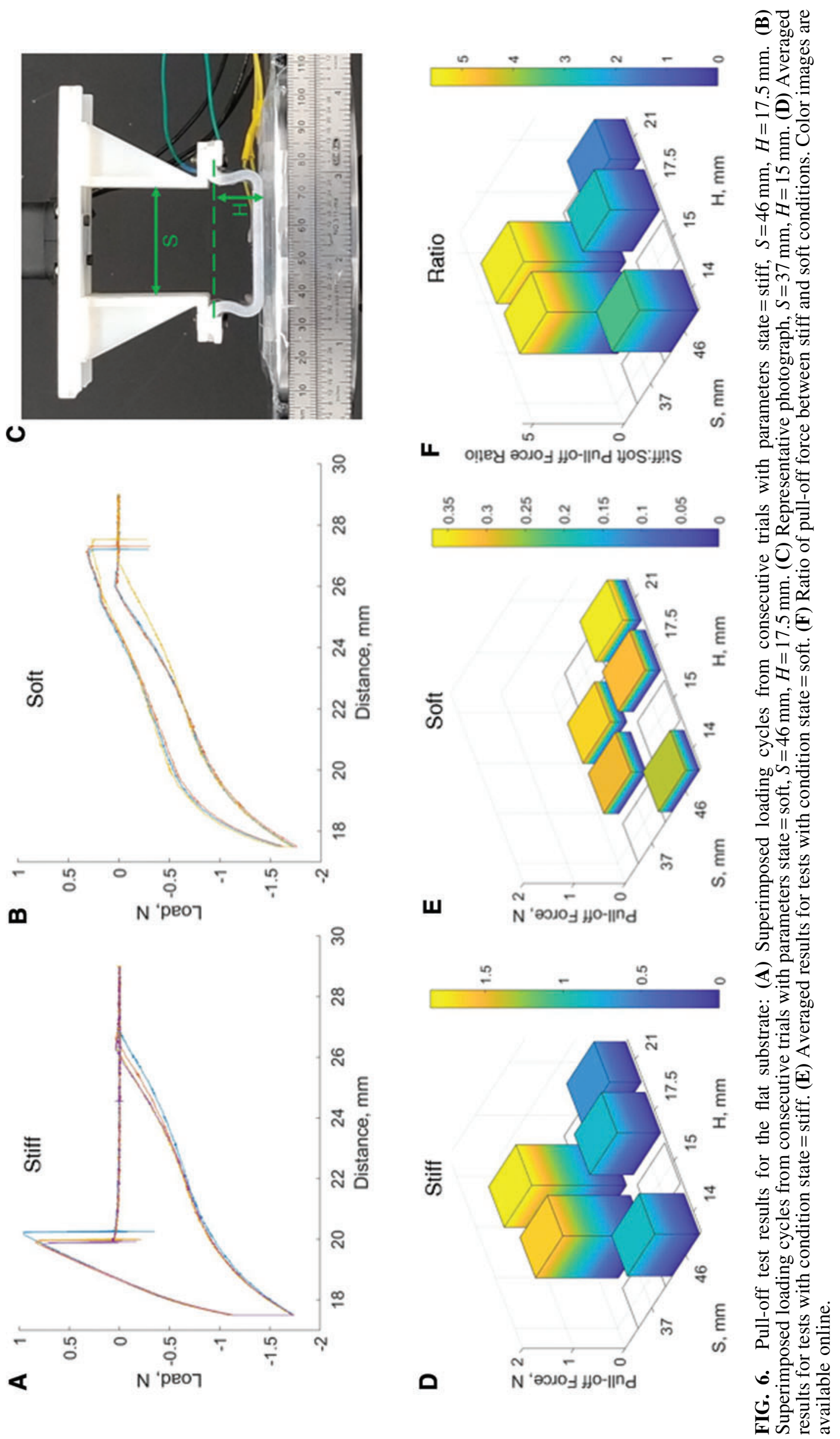

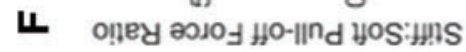

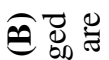

غ่

约政

드응 ॥

घำ

है⿲:

II

荨电

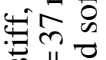

\|॥

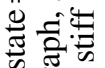

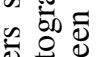

¿

है

记

产焉离

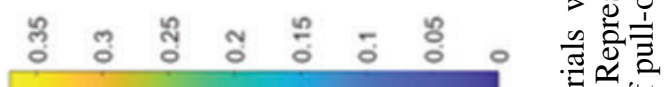

$\exists$ Oे

约

范

证

ठ도․

a

ㄴ. II

纪泀

잉.

ond :

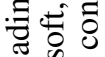

의

证

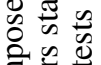

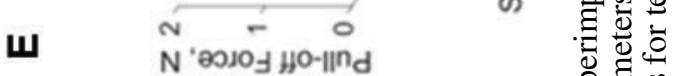

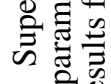



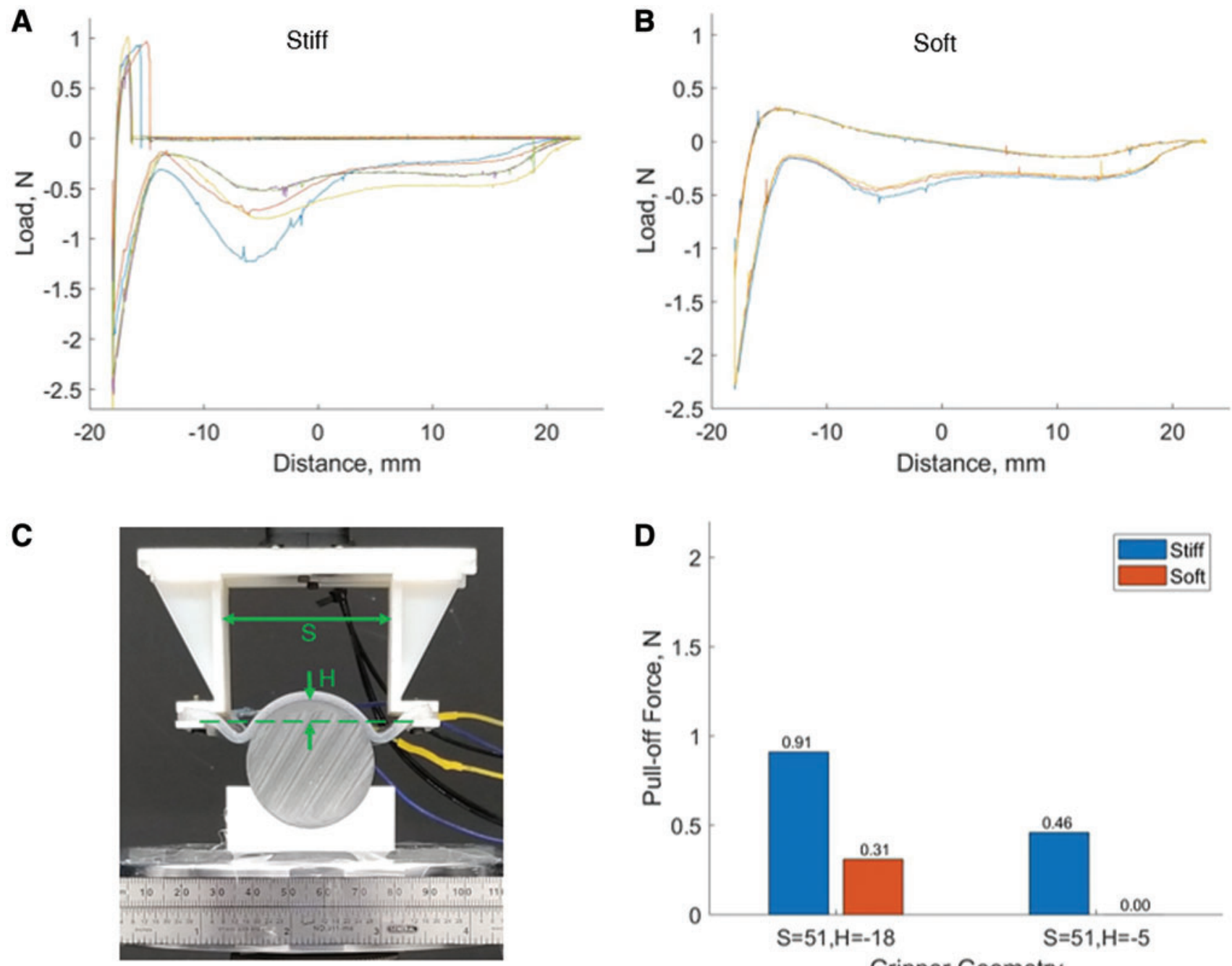

Gripper Geometry

FIG. 7. Pull-off test results for the cylindrical substrate: (A) Superimposed loading cycles from consecutive trials with parameters state $=$ stiff, $S=51 \mathrm{~mm}, H=-18 \mathrm{~mm}$. (B) Superimposed loading cycles from consecutive trials with parameters state $=$ soft, $S=51 \mathrm{~mm}, H=-18 \mathrm{~mm}$. (C) Representative photograph, $S=51 \mathrm{~mm}, H=-5 \mathrm{~mm}$. (D) Averaged results, $S$ held constant at $51 \mathrm{~mm}$. Color images are available online.

Beginning with the flat substrate results shown in Figure 6, it is evident that pull-off force increases when the contact pad is in its stiff state, rather than its soft state, before retraction. The increase in pull-off force as a result of this stiffness change was $\sim 2-6 \times$, depending on gripper geometry. This finding is consistent with the results of other studies that explored stiffness modulation for tunable adhesion. ${ }^{18,26}$

We also see that the pull-off forces corresponding to soft state tests are approximately constant regardless of gripper geometry. This result can be explained by the fact that the deformed shape corresponding to some combination of $S$ and $H$ values before retraction is not maintained during retraction without a stiffening of the core to "lock in" the shape. Instead, as the Instron crosshead is retracted, the nonadhered regions of the contact pad straighten and the pad gradually peels from the substrate. Still, the clamping distance $S$ will affect the peeling angle and thus the peel strength. However, this did not result in any significant change in pull-off force.

However, varying $S$ and $H$ did have a significant influence on pull-off force during stiff state tests. In the stiff state, the deformed shape before retraction is locked in. Furthermore, unlike in the soft state, the contact area between the gripper and the substrate just before pull-off is virtually the same as the original contact area. Without peeling, the pull-off force is governed by the contact area and the normal adhesion strength, which is influenced by load sharing on the interface. In general, stiffer materials allow for improved load sharing and, thus, adhesion enhancement. Similarly, the gripper geometry can affect the stress distribution on the interface, which can result in changes in pull-off force. The results show that the pull-off force increases with decreasing $H$ (for $S=46 \mathrm{~mm}$ ) and with decreasing $S$, mainly due to increases in contact area. For $S=37 \mathrm{~mm}$, the pull-off force decreases with decreasing $H$. In this case, the contact area decreases, so the increase in pull-off force could possibly be attributed to a change in the interfacial stress distribution resulting from the change in geometry. However, these results are based on a limited amount of data, and more testing will be required to arrive at a definitive conclusion regarding the impact of $S$ and $H$ on pull-off force. 
When analyzing results from tests with the cylindrical substrate in Figure 7, it is evident that the trend of increased pull-off force for stiff versus soft state tests still holds. As with the flat substrate, we observe that as $S$ is held constant and $H$ increases, the pull-off force decreases. The magnitudes of the pull-off force are also similar to what was observed with the flat substrate.

One possible source of uncertainty from the pull-off testing is that the contact pad was prone to experiencing small amounts of plastic deformation during testing. We believe that this was due to the fact that the flexible heater did not cover the entire area of the composite's surface (Fig. 3A). Consequently, the edges of the composite (not covered by the heater) were not always raised to the same temperature as the rest of the composite, which prevented them from fully softening and caused them to retain a small amount of curvature as the contact pad was continuously stretched (flattened) and compressed (curved) over the course of many loading cycles.

\section{Comparison of FEA and experimental results}

Using the finite element model described in the Finite Element Model section, the gripper's deformed profile after contact formation was examined for several combinations of $S$ and $H$. Generally, the predicted profiles show good agreement with the experimental profiles, as exemplified by the profiles shown in Figure 8.

To estimate the ratio of stiff to soft pull-off forces, the system compliance was determined for sets of $S$ and $H$ values in both the stiff and soft states. For all combinations of $S$ and $H$ considered, use of Equation (1) yielded a stiff to soft pull-off force ratios ranging from $\sim 8$ to 13 , as shown in Supplementary Figure S1. From a compliance perspective, and considering the $250 \times$ increase in the Young modulus of the core material upon stiffening, the theoretical maximum stiff to soft pull-off force ratio is expected to be in the range of $\sim \sqrt{250}$. This suggests a $16 \times$ increase in pull-off force, which is comparable to the range predicted by FEA. These predicted ratios are larger than those given by the experimental data, which shows up to a $6 \times$ increase in pull-off force from stiffening the core. The difference between the FEA prediction and the experimental results could be attributed to time-dependent effects, plastic deformations of the PCL core, and misalignment between the gripper and substrate in the experiments that would reduce the measured pull-off force, which are not captured by the FEA.

\section{Pick-and-place demonstrations}

In addition to pull-off testing, the gripper's efficacy was also evaluated based on pick-and-place demonstrations conducted with various objects. Objects successfully grasped and released included a playing card $(1.62 \mathrm{~g})$, a silicon wafer $(9.38 \mathrm{~g})$, a small tomato $(25.3 \mathrm{~g})$, and a juice can $(36.6 \mathrm{~g})$, as shown in Figure 9. Footage of these pick-and-place demonstrations is available in Supplementary Video S3-S6. See Supplementary Figure $\mathrm{S} 2$ for a block diagram detailing the control sequence for pick-and-place tasks.

In general, we found that this gripper excels at grasping flat smooth objects, which can be challenging for other grippers that grasp objects via force closure. It was even capable of lifting a small dinner plate weighing $147 \mathrm{~g}(1.44 \mathrm{~N})$, corresponding to $78 \%$ of the maximum pull-off force measured during pull-off testing (see Supplementary Video S7). The gripper is also capable of grasping objects with convex surfaces of various shapes and sizes, including a tomato and a juice can, as shown in Figure 9. Although this capability is generally limited to objects with simple rounded surfaces, it serves to demonstrate the gripper's versatility. After grasping, each of the objects was released and returned to near its original location by simply heating the contact pad and allowing the object to drop due to the decreased adhesion force.

In addition to flat and convex objects, the gripper geometry can be controlled before contact to allow for conformation to concave objects. To demonstrate this, the finite element model was used to predict the deformed gripper geometry when decreasing $S$ before contact. As shown in Supplementary
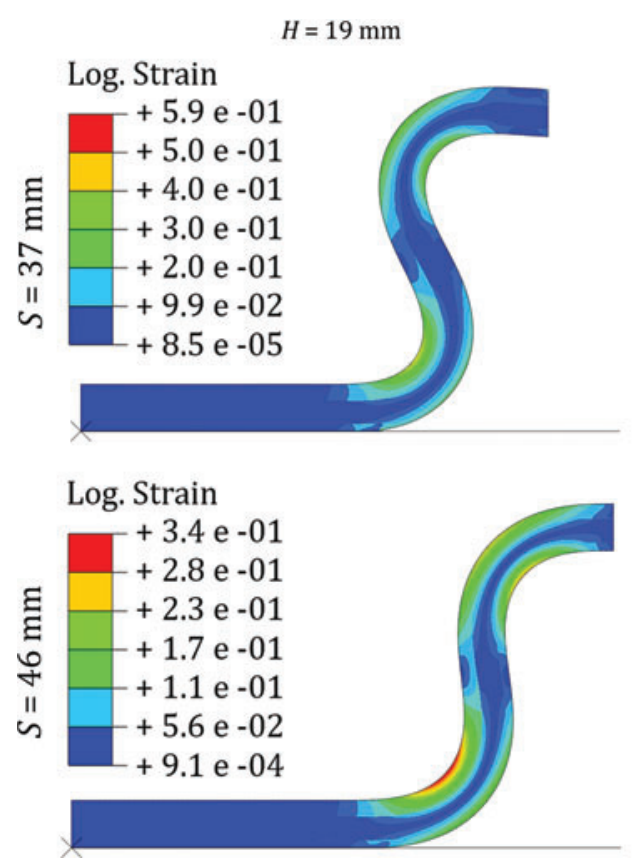
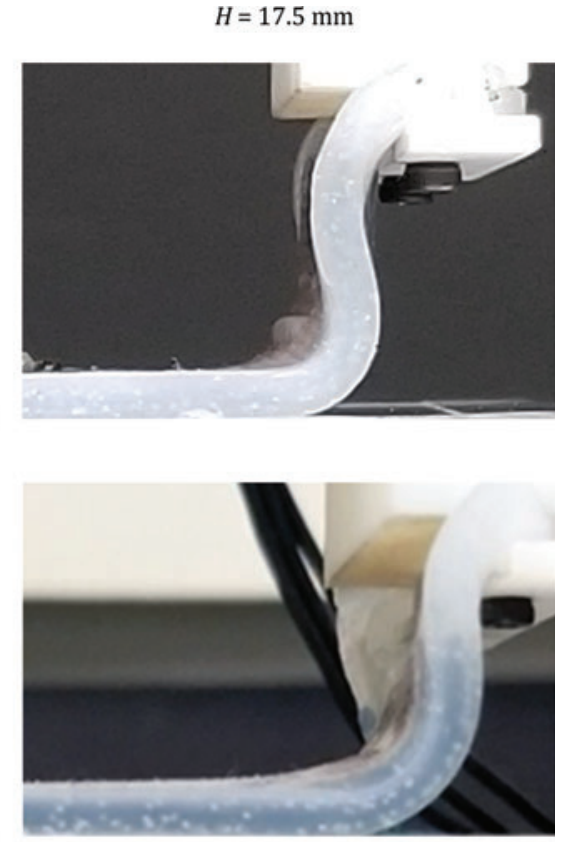

FIG. 8. Deformed profiles of the contact pad before retraction. Predictions from FEA with logarithmic maximum principal strain contours are shown in the left column, and experimental results are shown in the right column. FEA, finite element analysis. Color images are available online. 


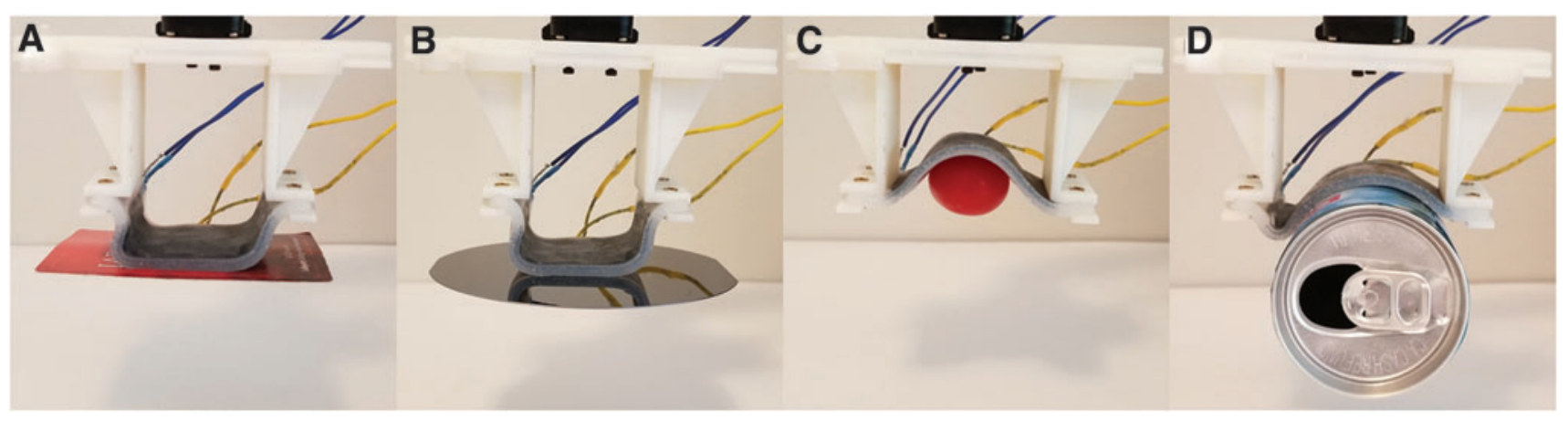

FIG. 9. Grasping various objects: (A) Playing card, 1.62 g. (B) Silicon wafer, 9.38 g. (C) Small tomato, 25.3 g. (D) Juice can, $36.6 \mathrm{~g}$. Color images are available online.

Figure S3, the radius of curvature and span of the bottom of the gripper can be significantly reduced by decreasing $S$. Therefore, the gripper can accommodate a range of concave objects, which is limited by the combination of object curvature and depth. Additionally, the peel angle will be smaller when grasping a concave object compared with a flat object and will continue to decrease with increasing object curvature. Since pull-off force increases with decreasing peel angle as suggested by the Kendall equation, ${ }^{46}$ we expect the load capacity of the gripper to increase when grasping concave objects.

The gripper also showed promise in handling fragile objects - such as a silicon wafer and a tomato-without causing any damage to them. This capability is a result of the low modulus of the gripper during contact formation $(0.09 \mathrm{MPa}$ for the outer Dragon Skin 10 layer and $2 \mathrm{MPa}$ for the softened PCL core) and the ability to form large contact areas. This results in low contact pressures that prevent object damage. The mean contact pressure exerted by the gripper can be estimated from the experimental data by dividing the preload (measured at Step 3 in Fig. 4A, B) by the contact area, which can be estimated using photographs from the experiments. Using this approach, we found that the largest mean contact pressure exerted by the gripper on the cylindrical substrate was $1 \mathrm{kPa}$ (see Supplementary Table S1 for additional data relating to estimation of contact pressures). For grasping of round fragile objects such as fruits and vegetables, this pressure is well below the threshold for causing damage. For example, a study by Pettersson et al. ${ }^{10}$ found that strawberries could withstand up to $6 \mathrm{kPa}$ without bruising.

Taken as a whole, these pick-and-place demonstrations are a validation of the gripper's applicability toward realworld tasks. Although there are several classes of objects which the gripper cannot reliably grasp-including those with rough or wet surfaces (which are problematic for many grippers that rely upon dry adhesion) or complex surface geometries - it exhibits an impressive degree of versatility given its simplicity. Importantly, because of the gripper's simplicity-specifically the fact that it requires only a single servo motor for actuation and a $9 \mathrm{~V}$ power supply to power the flexible heater-it can be easily integrated with most robotic systems, including the small uArm robot that was used for these demonstrations.

\section{Limitations}

While the gripper developed for this study has demonstrated its ability to successfully grasp and release various objects, it does have some limitations. First is the gripper's cycle time. Since the gripper's stiffness tuning capability is dependent on a thermally induced phase change, the amount of time required to perform a successful grasp is relatively large. Quantitatively, the gripper requires on average $3.3 \mathrm{~min}$ to heat from room temperature to $T_{\text {set }}=50^{\circ} \mathrm{C}$ and $4.5 \mathrm{~min}$ to cool to $\tilde{T}_{\text {stiff }}=35^{\circ} \mathrm{C}$ (as established in the Material Selection, Synthesis, and Characterization Section), resulting in an overall cycle time of $8.8 \mathrm{~min}$ to grasp a single object. This issue is common among stiffness tuning technologies that rely upon thermal activation. However, there are several steps that could be taken in future work to reduce this cycle time. One solution is to load the contact pad's silicone matrix with liquid metal to increase its thermal conductivity, as established by Bartlett et al. ${ }^{47}$ Another solution is to implement "vascular" cooling by running cold fluid through the contact pad while it cools, as demonstrated in multiple studies. ${ }^{17,20,48}$ Additionally, the PCL composite could be replaced with a material with a lower melting point and higher thermal conductivity.

Another limitation of the current gripper is that it is not always successful in releasing objects after grasping. In principle, when the gripper is ready to release an object, the contact pad can be heated to $T_{\text {set }}=50^{\circ} \mathrm{C}$ to reduce the adhesion strength. However, if the object is too light such that its weight is not sufficient to overcome the minimum adhesion strength, the object will not be released. In the case of light objects, it is useful to control $S$ and $H$ so as to form only the minimum amount of contact area required to lift the object. Then, if the object will not release, the distance between gripper jaws can be modulated to help facilitate peeling and object detachment, although this is not always successful. Once the contact pad is fully softened, the minimum adhesion strength ultimately depends on the surface energy of the contacting surfaces. Therefore, if release of very light objects is desired, a material with lower surface energy can be used for the outer surface of the contact pad.

\section{Conclusion}

This research focused on the development of a novel soft robotic gripper that relies upon stiffness and adhesion tuning capabilities enabled by controlling the temperature of a thermoplastic composite embedded in a soft contact pad. Pull-off tests performed using the gripper showed that stiffness tuning leads to enhanced adhesion and provided insight into the effect of gripper geometry and substrate geometry on 
adhesion strength. A finite element model was also developed to simulate the gripping procedure, which provided insight into the deformation and adhesion mechanics of the system. Finally, the gripper was integrated with a four DOF robotic arm and used to successfully grasp and release objects of various shapes, weights, and sizes.

Overall, this work demonstrates the efficacy of a stiffness tuning gripper for adaptive and controllable adhesion-based grasping. Our gripper represents an important milestone toward the development of a practical and versatile gripper enabled by stiffness and adhesion tuning capabilities. In future work, we hope to address some of the major limitations of the gripper, including its large cycle time and inconsistency in releasing objects, described in the Limitations section. There are also several possible extensions to this work, which we believe are promising. These include enabling magnetic actuation of the composite by loading it with magnetic particles; applying kirigami principles ${ }^{49}$ to achieve enhanced adhesion; and using polymer-based stiffness tuning elements for different applications, such as endoscopic surgery.

\section{Author Disclosure Statement}

No competing financial interests exist.

\section{Funding Information}

This work was funded by the NSF National Robotics Initiative by Awards 1830362 and 1830475 . Additionally, this work used the Extreme Science and Engineering Discovery Environment (XSEDE), which is supported by the National Science Foundation grant number ACI-1548562. ${ }^{50}$ Specifically, we used the Comet cluster provided by the San Diego Supercomputer Center (SDSC) at the University of California San Diego under allocation TG-MSS190018.

\section{Supplementary Material}

Supplementary Table S1

Supplementary Figure S1

Supplementary Figure S2

Supplementary Figure S3

Supplementary Video S1

Supplementary Video S2

Supplementary Video S3

Supplementary Video S4

Supplementary Video S5

Supplementary Video S6

Supplementary Video S7

\section{References}

1. Manti M, Cacucciolo V, Cianchetti M. Stiffening in soft robotics: a review of the state of the art. IEEE Rob Autom Mag 2016;23:93-106.

2. Wang L, Yang Y, Chen Y, et al. Controllable and reversible tuning of material rigidity for robot applications. Mater Today 2018;21:563-576.

3. Shintake J, Cacucciolo V, Floreano D, et al. Soft robotic grippers. Adv Mater 2018;30:1707035.

4. Brown E, Rodenberg N, Amend J, et al. Universal robotic gripper based on the jamming of granular material. Proc Natl Acad Sci U S A 2010;107:18809-18814.
5. Amend JR, Brown E, Rodenberg N, et al. A positive pressure universal gripper based on the jamming of granular material. IEEE Trans Robot 2012;28:341-350.

6. Cianchetti M, Ranzani T, Gerboni G, et al. Stiff-flop surgical manipulator: mechanical design and experimental characterization of the single module. In: IEEE/RSJ international conference on intelligent robots and systems. IEEE, 2013, pp. 3576-3581.

7. Wei Y, Chen Y, Ren T, et al. A novel, variable stiffness robotic gripper based on integrated soft actuating and particle jamming. Soft Robot 2016;3:134-143.

8. Mizushima K, Oku T, Suzuki Y, et al. Multifingered robotic hand based on hybrid mechanism of tendon-driven and jamming transition. In: IEEE International Conference on Soft Robotics (RoboSoft). IEEE, 2018, pp. 376-381.

9. Cao C, Zhao X. Tunable stiffness of electrorheological elastomers by designing mesostructures. Appl Phys Lett 2013;103:041901.

10. Pettersson A, Davis S, Gray JO, et al. Design of a magnetorheological robot gripper for handling of delicate food products with varying shapes. J Food Eng 2010;98:332338.

11. Eisenhaure JD, Xie T, Varghese S, et al. Microstructured shape memory polymer surfaces with reversible dry adhesion. ACS Appl Mater Interfaces 2013;5:7714-7717.

12. McCoul D, Rosset S, Besse N, et al. Multifunctional shape memory electrodes for dielectric elastomer actuators enabling high holding force and low-voltage multisegment addressing. Smart Mater Struct 2016;26:025015.

13. Wang W, Ahn S-H. Shape memory alloy-based soft gripper with variable stiffness for compliant and effective grasping. Soft Robot 2017;4:379-389.

14. Buckner TL, White EL, Yuen MC, et al. A move-and-hold pneumatic actuator enabled by self-softening variable stiffness materials. In: IEEE/RSJ International Conference on Intelligent Robots and Systems (IROS). IEEE, 2017, pp. 3728-3733.

15. Yang Y, Chen Y, Li Y, et al. Novel variablestiffness robotic fingers with built-in position feedback. Soft Robot 2017;4:338-352.

16. Firouzeh A, Salerno M, Paik J. Stiffness control with shape memory polymer in underactuated robotic origamis. IEEE Trans Robot 2017;33:765-777.

17. Hao Y, Liu Z, Liu J, et al. A soft gripper with programmable effective length, tactile and curvature sensory feedback. Smart Mater Struct 2020;29:035006.

18. Krahn J, Sameoto D, Menon C. Controllable biomimetic adhesion using embedded phase change material. Smart Mater Struct 2010;20:015014.

19. Cheng NG, Gopinath A, Wang L, et al. Thermally tunable, self-healing composites for soft robotic applications. Macromol Mater Eng 2014;299:1279-1284.

20. Balasubramanian A, Standish M, Bettinger CJ. Microfluidic thermally activated materials for rapid control of macroscopic compliance. Adv Funct Mater 2014;24:4860-4866.

21. Wang L, Culha U, Iida F. A dragline-forming mobile robot inspired by spiders. Bioinspiration Biomimetics 2014;9: 016006.

22. Andy McEvoy M, Correll N. Thermoplastic variable stiffness composites with embedded, networked sensing, actuation, and control. J Compos Mater 2015;49:1799-1808.

23. Rich S, Jang S-H, Park Y-L, et al. Liquid metal-conductive thermoplastic elastomer integration for low-voltage stiffness tuning. Adv Mater Technol 2017;2:1700179. 
24. Shan W, Diller S, Tutcuoglu A, et al. Rigidity-tuning conductive elastomer. Smart Mater Struct 2015;24:065001.

25. Mohammadi Nasab A, Sabzehzar A, Tatari M, et al. A soft gripper with rigidity tunable elastomer strips as ligaments. Soft Robot 2017;4:411-420.

26. Tatari M, Mohammadi Nasab A, Turner KT, et al. Dynamically tunable dry adhesion via subsurface stiffness modulation. Adv Mater Interfaces 2018;5:1800321.

27. Rich SI, Nambeesan V, Khan R, et al. Tuning the composition of conductive thermoplastics for stiffness switching and electrically activated healing. J Intell Mater Syst Struct 2019;30:2908-2918.

28. Shan W, Lu T, Majidi C. Soft-matter composites with electrically tunable elastic rigidity. Smart Mater Struct 2013; 22:085005.

29. Schubert BE, Floreano D. Variable stiffness material based on rigid low-meltingpoint-alloy microstructures embedded in soft poly (dimethylsiloxane) (PDMS). RSC Adv 2013;3: 24671-24679.

30. Shintake J, Schubert B, Rosset S, et al. Variable stiffness actuator for soft robotics using dielectric elastomer and low-melting-point alloy. In: IEEE/RSJ International Conference on Intelligent Robots and Systems (IROS). IEEE, 2015, pp. 1097-1102.

31. Van Meerbeek IM, Mac Murray BC, Kim JW, et al. Morphing metal and elastomer bicontinuous foams for reversible stiffness, shape memory, and self-healing soft machines. Adv Mater 2016;28:2801-2806.

32. Sameoto D, Ferguson B. Robust large-area synthetic dry adhesives. J Adhes Sci Technol 2014;28:337-353.

33. Song S, Majidi C, Sitti M. Geckogripper: a soft, inflatable robotic gripper using gecko-inspired elastomer microfiber adhesives. In: IEEE/RSJ International Conference on Intelligent Robots and Systems. IEEE, 2014, pp. 4624 4629.

34. Song S, Drotlef D-M, Majidi C, et al. Controllable load sharing for soft adhesive interfaces on three-dimensional surfaces. Proc Natl Acad Sci U S A 2017;114:E4344E4353.

35. Glick P, Suresh SA, Ruffatto D, et al. A soft robotic gripper with gecko-inspired adhesive. IEEE Robot Autom Lett 2018;3:903-910.

36. Shintake J, Rosset S, Schubert B, et al. Versatile soft grippers with intrinsic electroadhesion based on multifunctional polymer actuators. Adv Mater 2016;28:231-238.

37. Hughes J, Iida F. Tack and deformation based sensorised gripping using conductive hot melt adhesive. In: IEEE International Conference on Soft Robotics (RoboSoft). IEEE, 2018, pp. 553-558.
38. Testa P, Chappuis B, Kistler S, et al. Switchable adhesion of soft composites induced by a magnetic field. Soft Matter 2020;16:5806-5811.

39. Croll AB, Hosseini N, Bartlett MD. Switchable adhesives for multifunctional interfaces. Adv Mater Technol 2019;4: 1900193

40. Ye Z, Lum GZ, Song S, et al. Phase change of gallium enables highly reversible and switchable adhesion. Adv Mater 2016;28:5088-5092.

41. Song S, Drotlef D-M, Paik J, et al. Mechanics of a pressurecontrolled adhesive membrane for soft robotic gripping on curved surfaces. Extreme Mech Lett 2019;30:100485.

42. Bartlett MD, Markvicka EJ, Majidi C. Rapid fabrication of soft, multilayered electronics for wearable biomonitoring. Adv Funct Mater 2016;26:8496-8504.

43. Markvicka E, Wang G, Lee Y-C, et al. Electrodermis: fully untethered, stretchable, and highly-customizable electronic bandages. In: Proceedings of the 2019 CHI Conference on Human Factors in Computing Systems. 2019:1-10.

44. Mengüç Y, Röhrig M, Abusomwan U, et al. Staying sticky: contact self-cleaning of gecko-inspired adhesives. J R Soc Interface 2014;11:20131205.

45. Bartlett MD, Crosby AJ. Scaling normal adhesion force capacity with a generalized parameter. Langmuir 2013;29: 11022-11027.

46. Kendall K. The adhesion and surface energy of elastic solids. J Phys D Appl Phys 1971;4:1186.

47. Bartlett MD, Kazem N, Powell-Palm MJ, et al. High thermal conductivity in soft elastomers with elongated liquid metal inclusions. Proc Natl Acad Sci U S A 2017; 114:2143-2148.

48. He Q, Wang Z, Song Z, et al. Bioinspired design of vascular artificial muscle. Adv Mater Technol 2019;4:1800244.

49. Hwang D-G, Trent K, Bartlett MD. Kirigami-inspired structures for smart adhesion. ACS Appl Mater Interfaces 2018;10:6747-6754.

50. Towns J, Cockerill T, Dahan M, et al. XSEDE: accelerating scientific discovery. Comput Sci Eng 2014;16:62-74.

Address correspondence to: Carmel Majidi

Department of Mechanical Engineering and Robotics Institute

Carnegie Mellon University 5000 Forbes Avenue Pittsburgh, PA 15213

USA

E-mail: cmajidi@andrew.cmu.edu 